\title{
NOMINATA DE PARECERISTAS
}

Dossiê Diálogos pandêmicos no ensino, na pesquisa e na formação de professores de línguas e literaturas

Número 20 (Jan.-Abr. 2021)

Adriana Lúcia de Escobar Chaves de Barros Universidade Estadual de Mato Grosso do Sul

Andréa Rodrigues

Universidade do Estado do Rio de Janeiro

Isabel Cristina Moraes Bezerra

Universidade do Estado do Rio de Janeiro

Marcia Lisbôa Costa de Oliveira

Universidade do Estado do Rio de Janeiro

Maria Betânia Almeida Pereira

Universidade do Estado do Rio de Janeiro

Mônica Gomes da Silva

Universidade Federal do Recôncavo da Bahia

Simone Batista da Silva

Universidade Federal Rural do Rio de Janeiro 